\title{
Hybrid mesons from lattice QCD
}

\author{
Colin Morningstar \\ Florida International University, Miami, Florida, USA
}

\begin{abstract}
Recent lattice simulation studies of heavy-quark hybrid mesons in which the quark and antiquark are bound together by an excited gluon field are summarized.
\end{abstract}

Hybrid mesons are states in which a valence quark and an antiquark are bound by an excited gluon field. Interest in such states has been recently sparked by observations of resonances with exotic $1^{-+}$quantum numbers[1]. In fact, the proposed Hall D at Jefferson Lab will be devoted to the search for hybrid mesons. Although our understanding of these states remains meager, recent lattice simulations have shed some light on their nature. In this talk, I focus on studies of hybrid mesons containing heavy quarks, although I conclude with brief comments on light-quark hybrid-meson simulations.

One expects that a heavy-quark meson can be treated similar to a diatomic molecule: the slow valence heavy quarks correspond to the nuclei and the fast gluon and light sea quark fields correspond to the electrons[2]. First, the quark $Q$ and antiquark $\bar{Q}$ are treated as static color sources and the energy levels of the fast degrees of freedom are determined as a function of the $Q \bar{Q}$ separation $r$, each such energy level defining an adiabatic surface or potential. The motion of the slow heavy quarks is then described in the leading Born-Oppenheimer (LBO) approximation by the Schrödinger equation using each of these potentials. Conventional quarkonia are based on the lowest-lying potential; hybrid quarkonium states emerge from the excited potentials.

The validity of such a simple physical picture relies on the smallness of higherorder spin, relativistic, and retardation effects and mixings between states based on different adiabatic surfaces. Recently, it was demonstrated that retardation and mixing effects do not spoil the leading Born-Oppenheimer approximation for the (quenched) low-lying conventional and hybrid spectrum[3]. In Ref. [4], the introduction of the heavy-quark spin was shown to lead to significant level shifts but not to significant mixings between states based on different potentials. In this talk, the leading Born-Oppenheimer approximation is described, the findings of Refs. [3] and [4] are summarized, and the 

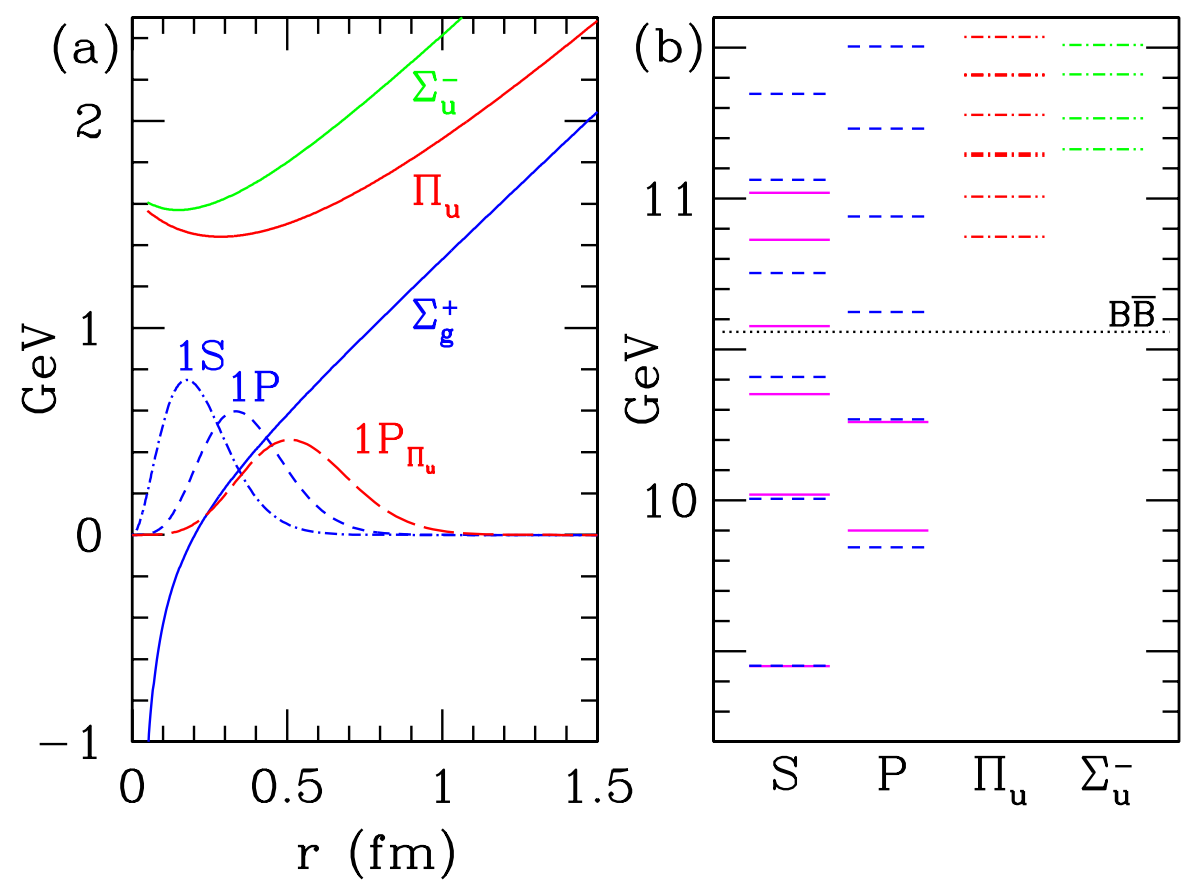

Fig. 1. (a) Static potentials and radial probability densities against quark-antiquark separation $r$ for $r_{0}^{-1}=450 \mathrm{MeV}$. (b) Spin-averaged $\bar{b} b$ spectrum in the LBO approximation (light quarks neglected). Solid lines indicate experimental measurements. Short dashed lines indicate the $S$ and $P$ state masses obtained using the $\Sigma_{g}^{+}$potential with $M_{b}=4.58 \mathrm{GeV}$. Dashed-dotted lines indicate the hybrid quarkonium states obtained from the $\Pi_{u}(L=1,2,3)$ and $\Sigma_{u}^{-}(L=0,1,2)$ potentials.

expected (but currently unknown) impact of sea quark effects on the LBO spectrum calculation is discussed.

The spectrum of the fast gluon field in the presence of a static quark-antiquark pair has been determined in lattice studies[5]. The three lowest-lying levels are shown in Fig. 1. Due to computational limitations, sea quark effects have been neglected in these calculations; their expected impact on the hybrid meson spectrum will be discussed later. The levels in Fig. 1 are labeled by the magnitude $\Lambda$ of the projection of the total angular momentum $\mathbf{J}_{g}$ of the gluon field onto the molecular axis, and by $\eta= \pm 1$, the symmetry under the charge conjugation combined with spatial inversion about the midpoint between the $Q$ and $\bar{Q}$. States with $\Lambda=0,1,2, \ldots$ are denoted by $\Sigma, \Pi, \Delta, \ldots$, respectively. States which are even (odd) under the above-mentioned $C P$ operation are denoted by the subscripts $g(u)$. An additional \pm superscript for the $\Sigma$ states refers to even or odd symmetry under a reflection in a plane containing the molecular axis. The potentials are calculated in terms of the hadronic scale parameter $r_{0}$; in Fig. 1, $r_{0}^{-1}=450 \mathrm{MeV}$ has been assumed.

The LBO spectrum is obtained by solving the radial Schrödinger equation. Results for the LBO spectrum of conventional $\bar{b} b$ and hybrid $\bar{b} g b$ states are shown in Fig. 1. Below the $\bar{B} B$ threshold, the LBO results are in very good agreement 


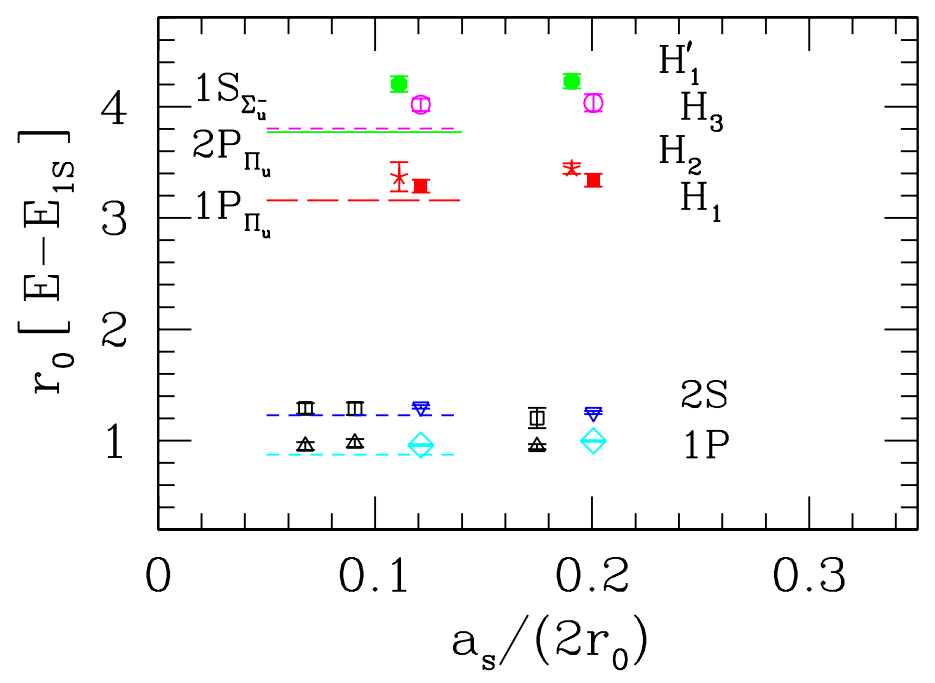

Fig. 2. Simulation results from Ref. [3] for the level splittings (in terms of $r_{0}$ and with respect to the $1 S$ state) against the lattice spacing $a_{s}$. Results from Ref. [6] using an NRQCD action with higher-order corrections are shown as $\square$ and $\triangle$. The horizontal lines show the LBO predictions.

with the spin-averaged experimental measurements of bottomonium states. Above the threshold, agreement with experiment is lost, suggesting significant corrections either from mixing and other higher-order effects or (more likely) from light sea quark effects. Note from the radial probability densities shown in Fig. 1 that the size of the hybrid state is large in comparison with the conventional $1 S$ and $1 P$ states.

The importance of retardation and leading-order mixings between states based on different adiabatic potentials can be tested by comparing the LBO level splittings with those determined from meson simulations using a leading-order non-relativistic (NRQCD) heavy-quark action. Such a test was carried out in Ref. [3]. The NRQCD action included only a covariant temporal derivative and the leading kinetic energy operator (with two other operators to remove lattice spacing errors). The only difference between the leading Born-Oppenheimer Hamiltonian and the lowest-order NRQCD Hamiltonian was the $\boldsymbol{p} \cdot \boldsymbol{A}$ coupling between the quark color charge in motion and the gluon field. The level splittings (in terms of $r_{0}$ and with respect to the $1 S$ state) of the conventional $2 S$ and $1 P$ states and four hybrid states were compared (see Fig. 2) and found to agree within $10 \%$, strongly supporting the validity of the leading BornOppenheimer picture. The meson operators used are listed in Table 1 and are described in much more detail in Ref. [3].

The question of whether or not quark spin interactions spoil the validity of the Born-Oppenheimer picture for heavy-quark hybrids has been addressed in Ref. [4]. Simulations of several hybrid mesons using an NRQCD action including the spin interaction $-c_{1} \boldsymbol{\sigma} \cdot \boldsymbol{B} / 2 M_{b}$ and neglecting light sea quark effects were carried out; the resulting splittings are shown in Fig. 3 for various values 
Table 1

\begin{tabular}{|c|l|c|c|}
\hline$J^{P C}$ & & Degeneracies & Operator \\
\hline \hline $0^{-+}$ & $S$ wave & $1^{--}$ & $\tilde{\chi}^{\dagger}\left[\tilde{\Delta}^{(2)}\right]^{p} \tilde{\psi}$ \\
$1^{+-}$ & $P$ wave & $0^{++}, 1^{++}, 2^{++}$ & $\tilde{\chi}^{\dagger} \tilde{\boldsymbol{\Delta}} \tilde{\psi}$ \\
$1^{--}$ & $H_{1}$ hybrid & $0^{-+}, 1^{-+}, 2^{-+}$ & $\tilde{\chi}^{\dagger} \tilde{\mathbf{B}}\left[\tilde{\Delta}^{(2)}\right]^{p} \tilde{\psi}$ \\
$1^{++}$ & $H_{2}$ hybrid & $0^{+-}, 1^{+-}, 2^{+-}$ & $\tilde{\chi}^{\dagger} \tilde{\mathbf{B}} \times \tilde{\boldsymbol{\Delta}} \tilde{\psi}$ \\
$0^{++}$ & $H_{3}$ hybrid & $1^{+-}$ & $\tilde{\chi}^{\dagger} \tilde{\mathbf{B}} \cdot \tilde{\boldsymbol{\Delta}} \tilde{\psi}$ \\
\hline
\end{tabular}

The meson operators used in Ref. [3]. Note that in the $0^{-+}$and $1^{--}$sectors, four operators were used by taking $p=0,1,2,3$. The corresponding $S=1$ states, degenerate for the spin-independent NRQCD action used, are also listed.

of the coupling $c_{1}$ (which is expected to be near unity) and are surprisingly large. However, the authors of Ref. [4] suggest that these splittings do not signal a breakdown of the Born-Oppenheimer picture. First, they claim that no significant mixing of their non-exotic $0^{-+}, 1^{--}$, and $2^{-+}$hybrid meson operators with conventional states was observed; unfortunately, this claim is not convincing since a correlation matrix analysis was not used and the effective masses associated with these correlators were not shown. Secondly, the authors argue that calculations using the bag model support their suggestion. Contributions to the spin splittings in Fig. 3 from the three diagrams shown in Fig. 4 were estimated in the bag model. Graph (a) is a weak effect (producing level shifts of order $1 \mathrm{MeV}$ or less) since it involves the $Q \bar{Q}$ wavefunction near the origin which is suppressed in hybrid states. Graph (b) is the dominant contribution (producing level shifts consistent with those shown in Fig. 3) since it depends on the quark wavefunction in the bulk; significant contributions from graph (b) do not necessarily spoil the Born-Oppenheimer picture. Graph (c) represents mixings between states based on different adiabatic surfaces and large contributions from such diagrams would signal a breakdown of the Born-Oppenheimer picture; however, bag model estimates of this mixing amplitude are very small of order $10^{-4}$. These facts are not conclusive evidence that heavy-quark spin effects do not spoil the Born-Oppenheimer picture, but they are highly suggestive.

The dense spectrum of hybrid states shown in Fig. 1 neglects the effects of light sea quark-antiquark pairs. In order to include these effects in the LBO, the adiabatic potentials must be determined fully incorporating the light quark loops. Such computations using lattice simulations are very challenging, and to date, high precision results for these potentials for separations out to $2 \mathrm{fm}$ are not yet available. For separations below $1 \mathrm{fm}$, the $\Sigma_{g}^{+}$and $\Pi_{u}$ potentials change very little[7] from the behavior shown in Fig. 1, suggesting that a few of the lowest-lying hybrid states may exist as well-defined resonances. However, for $Q \bar{Q}$ separations greater than $1 \mathrm{fm}$, the adiabatic surfaces should change dramatically from the behavior shown in Fig. 1; instead of increasing 


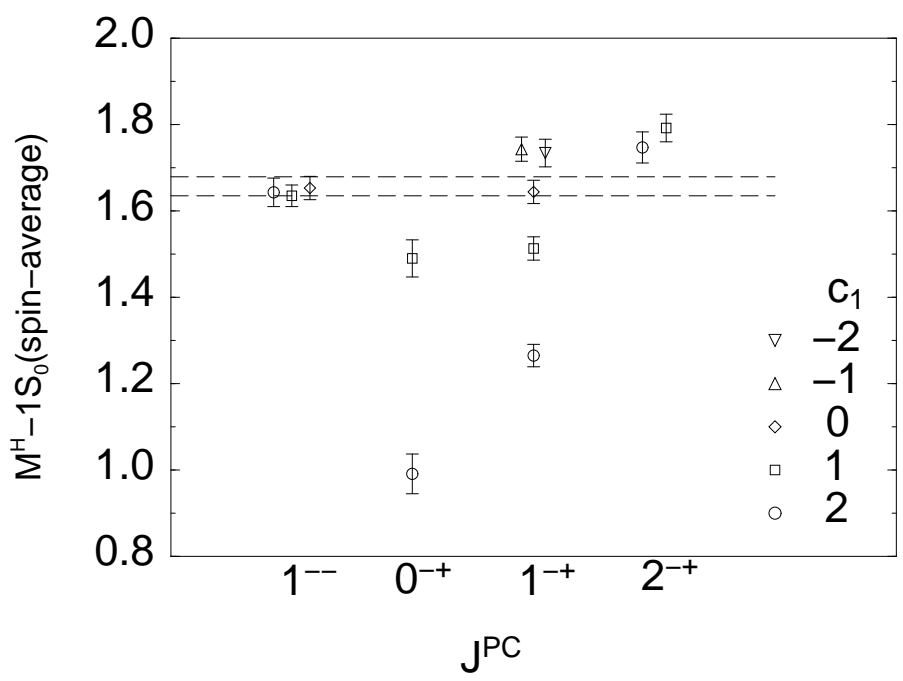

Fig. 3. Variation of the hybrid masses (in GeV) with $c_{1}$ (from Ref. [4]).

indefinitely, they should eventually level off since the static $\bar{Q} g Q$ state can undergo fission into two separate $\bar{Q} q$ color singlets, where $q$ is a light quark. The precise behavior of these potentials due to configuration mixing with $\bar{B} B$ mesons in the transition region is unknown; in fact, it is not even known if a fission barrier occurs. Clearly, such potentials cannot support the plethora of conventional and hybrid states shown in Fig. 1; the formation of bound states and resonances substantially extending over $1 \mathrm{fm}$ seems unlikely. Whether or not the light sea quark-antiquark pairs spoil the Born-Oppenheimer picture is currently unknown. Future unquenched simulations should help to answer this question. I remain hopeful that the simple physical picture provided by the Born-Oppenheimer expansion for both the low-lying conventional and hybrid heavy-quark mesons will survive the introduction of the light sea quark effects. I should mention once again that the discrepancies of the spin-averaged LBO predictions with experiment above the $B \bar{B}$ threshold seen in Fig. 1 are possibly caused by our neglect of light sea quark-antiquark pairs.

A summary of recent light-quark and charmonium $1^{-+}$hybrid mass calculations is presented in Table 2. With the exception of Ref. [11], all results neglect light sea quark loops. The introduction of two flavors of dynamical quarks in Ref. [11] yielded little change to the hybrid mass, but this finding should not be considered definitive due to uncontrolled systematics (unphysically large

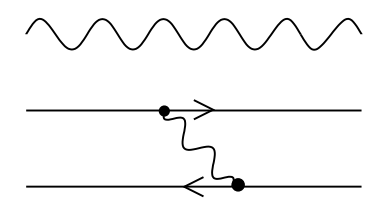

(a)

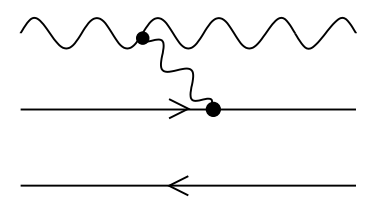

(b)

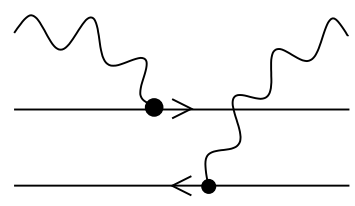

(c)

Fig. 4. Bag model diagrams studied in Ref. [4]. A wavy line indicates a gluon, and the two straight lines with arrows show the quark and antiquark. 


\begin{tabular}{|lc|c|l||ll|l|}
\hline \multicolumn{3}{|c||}{ Light quark $1^{-+}$} & \multicolumn{3}{c|}{ Charmonium $1^{-+}-1 S$} \\
\hline \multicolumn{2}{|c|}{ Ref. \& Method } & $N_{f}$ & $M(\mathrm{GeV})$ & \multicolumn{2}{c|}{ Ref. \& Method } & $\Delta M(\mathrm{GeV})$ \\
\hline \hline UKQCD 97[8] & SW & 0 & $1.87(20)$ & MILC 97[9] & W & $1.34(8)(20)$ \\
MILC 97[9] & W & 0 & $1.97(9)(30)$ & MILC 99[10] & SW & $1.22(15)$ \\
MILC 99[10] & SW & 0 & $2.11(10)$ & CP-PACS 99[12] & NR & $1.323(13)$ \\
LaSch 99[11] & W & 2 & $1.9(2)$ & JKM 99[3] & LBO & 1.19 \\
\hline
\end{tabular}

Table 2

Recent results for the light quark and charmonium $1^{-+}$hybrid meson masses. Method abbreviations: $\mathrm{W}=$ Wilson fermion action; $\mathrm{SW}=$ improved clover fermion action; $\mathrm{NR}=$ nonrelativistic heavy quark action. $N_{f}$ is the number of dynamical light quark flavors used.

quark masses, inadequate treatment of resonance properties in finite volume, etc.). All estimates of the light quark hybrid mass are near $2.0 \mathrm{GeV}$, well above the experimental candidates found in the range 1.4-1.6 GeV. Perhaps sea quark effects will resolve this discrepancy, or perhaps the observed states are not hybrids. Some authors have suggested that they may be four quark $\bar{q} \bar{q} q q$ states. Clearly, there is still much to be learned about these exotic QCD resonances.

\section{References}

[1] D. Thompson et al., Phys. Rev. Lett. 79, 1630 (1997); G. Adams et al., Phys. Rev. Lett. 81, 5760 (1998); A. Abele et al., Phys. Lett. B423, 175 (1998).

[2] P. Hasenfratz, R. Horgan, J. Kuti, J. Richard, Phys. Lett. B95, 299 (1980).

[3] K.J. Juge, J. Kuti, and C. Morningstar, Phys. Rev. Lett. 82, 4400 (1999).

[4] I. Drummond, N. Goodman, R. Horgan, H. Shanahan, and L. Storoni, Phys. Lett. B478, 151 (2000).

[5] K.J. Juge, J. Kuti, and C. Morningstar, Nucl. Phys. B(Proc. Suppl.) 63 326, (1998); and unpublished (hep-lat/9809098).

[6] C. Davies et al., Phys. Rev. D58, 054505 (1998).

[7] G. Bali, private communication, unpublished.

[8] P. Lacock et al., Phys. Lett. B401, 308 (1997).

[9] C. Bernard et al., Phys. Rev. D56, 7039 (1997).

[10] C. Bernard et al., Nucl. Phys. B(Proc. Suppl.) 73, 264 (1999).

[11] P. Lacock and K. Schilling, Nucl. Phys. B(Proc. Suppl.) 73, 261 (1999).

[12] T. Manke et al., Phys. Rev. Lett. 82, 4396 (1999). 